\title{
Correlation observables in $\Upsilon D$ pair production at the LHC within the parton Reggeization approach
}

A.V. Karpishkov ${ }^{1}$, M.A. Nefedov ${ }^{1,2}$, V.A. Saleev ${ }^{1, \pi}$

${ }^{1}$ Samara National Research University, Moskovskoe Shosse 34, 846064, Samara, Russia

${ }^{2}$ II. Institut für Theoretische Physik, Universität Hamburg, Luruper Chaussee 149, 22761

Hamburg, Germany

『E-mail: saleev@samsu.ru

\begin{abstract}
We study angular correlations in associated hadroproduction of $\Upsilon(1 S)$ with the $D^{ \pm}$and $D^{0}$ mesons at the LHC in the Leading Order of the parton Reggeization approach. Hadronization of $b \bar{b}$-pair to $\Upsilon(1 S)$ is described within the NRQCD-factorization framework. Production of $D$-mesons is described in the fragmentation model with scale-dependent fragmentaion functions. We have found good agreement with $\mathrm{LHCb}$ data for various differential distributions, except for the case of spectra on azimuthal angle differences at the small $\triangle \varphi$ values. The total cross-section in our Single Parton Scattering model, calculated under conservative assumptions, accounts for almost one half of observed cross-section, thus dramatically shrinking the room for Double Parton Scattering mechanism.
\end{abstract}

Keywords: LHC, quantum chromodynamics, parton Reggeization approach, angular correlations, $\Upsilon$ meson, $D$ meson, single parton scattering, double parton scattering.

PACS: 12.38.-t.

\section{Introduction}

Theoretical and experimental study of angular and momentum correlations in pair production of hadrons or jets in high energy hadronic collisions is very important task for various reasons. First, this is a challenging test of our understanding of higher-order corrections in quantum chromodynamics (QCD). In general it is a nontrivial task to provide reliable predictions for such multiscale and correlational observables, based on the conventional Collinear Parton Model (CPM) of QCD. Second, if heavy quarkonia are involved, various models of hadronization of heavy quark pair, such as NRQCD factorization formalism [1], can be tested. And last but not least, correlational observables in pair-production of jets, photons and heavy mesons has become a primary tool in experimental searches of manifestations of Double Parton Scattering(DPS) mechanism in proton-proton collisions at high energies, see e.g. [2, 3].

In the present contribution we discuss associated production of bottomonia and open charm hadrons in forward rapidity region in $p p$ collisions at the $\sqrt{S}=7$ and $8 \mathrm{TeV}$ which has been observed by the LHCb Collaboration [2]. Comparison of theoretical predictions obtained in leading order (LO) of CPM with the measured cross-sections and the differential distributions pointed towards DPS as the main production mechanism. However, recently [4], we have found that total cross sections and different spectra of same-sign $(D D)$ pairs at LHCb [3] can be described by the Single Parton Scattering (SPS) mechanism, if higher-order QCD corrections are approximately taken into account using the parton Reggeization approach (PRA) [5, 6]. The gauge-invariant amplitude of gluon pair production in the fusion of two off-shell(Reggeized) gluons together with scale-dependent $g \rightarrow D$ fragmentation functions where key ingredients of calculations in Ref. [4]. In the present note, we present the study of production of $\Upsilon(1 S) D^{0}$ and $\Upsilon(1 S) D^{+}$pairs in the combined approach based on PRA, nonrelativistic QCD (NRQCD) [1] factorization and fragmentation model.

\section{Parton Reggeization Approach}

The brief description of LO approximation of PRA is presented below. More details can be found in [6], the development of PRA in the NLO approximation is further discussed in [7]. The main ingre- 
dients of PRA are factorization formula, unintegrated parton distribution functions (unPDF's) and gauge-invariant amplitudes with off-shell initial-state partons, derived using the Lipatov's Effective Field Theory (EFT) of Reggeized gluons [8] and Reggeized quarks [9].

Factorization formula of PRA in LO approximation for the process $p+p \rightarrow \mathcal{Y}+X$, can be obtained from factorization formula of the collinear parton model (CPM) for the auxiliary hard subprocess $g+g \rightarrow g+\mathcal{Y}+g$. In the Ref. [6] the modified Multi-Regge Kinematics (mMRK) approximation for the auxiliary amplitude is constructed, which correctly reproduces the Multi-Regge and collinear limits of corresponding QCD amplitude. This mMRK-amplitude has t-channel factorized form, which allows one to rewrite the cross-section of auxiliary subprocess in a $k_{T}$-factorized form:

$$
d \sigma=\int_{0}^{1} \frac{d x_{1}}{x_{1}} \int \frac{d^{2} \mathbf{q}_{T 1}}{\pi} \tilde{\Phi}_{g}\left(x_{1}, t_{1}, \mu^{2}\right) \int_{0}^{1} \frac{d x_{2}}{x_{2}} \int \frac{d^{2} \mathbf{q}_{T 2}}{\pi} \tilde{\Phi}_{g}\left(x_{2}, t_{2}, \mu^{2}\right) \cdot d \hat{\sigma}_{\mathrm{PRA}},
$$

where $t_{1,2}=-\mathbf{q}_{T 1,2}^{2}$, the partonic cross-section $\hat{\sigma}_{\mathrm{PRA}}$ in PRA is determined by squared PRA amplitude, $\overline{\left|\mathcal{A}_{P R A}\right|^{2}}$. Despite the fact that four-momenta $\left(q_{1,2}\right)$ of partons in the initial state of $\mathcal{A}_{P R A}$ are off-shell $\left(q_{1,2}^{2}=-t_{1,2}<0\right)$, the PRA hard-scattering amplitude is gauge-invariant because the initial-state off-shell gluons are treated as Reggeized gluons $(R)$ in a sence of gauge-invariant EFT for QCD processes in Multi-Regge Kinematics(MRK), introduced by L.N. Lipatov in [8]. The Feynman rules of this EFT are written down in Ref. [10].

The tree-level "unintegrated PDFs" (unPDFs) $\tilde{\Phi}_{g}\left(x_{1,2}, t_{1,2}, \mu^{2}\right)$ in Eq. (1) are equal to the convolution of the collinear PDF $f_{g}\left(x, \mu^{2}\right)$ and DGLAP splitting function $P_{g g}(z)$ with the factor $1 / t_{1,2}$. Consequently, the cross-section (1) with such "unPDFs" contains the collinear divergence at $t_{1,2} \rightarrow 0$ and infrared (IR) divergence at $z_{1,2} \rightarrow 1$. To regularize the latter, we observe, that the mMRK expression gives a reasonable approximation for the exact matrix element only in the rapidity-ordered part of the phase-space $y_{g_{1}}>y_{\mathcal{Y}}>y_{g_{2}}$. From this requirement, the following cutoff on $z_{1,2}$ can be derived: $z_{1,2}<1-\Delta_{K M R}\left(t_{1,2}, \mu^{2}\right)$, where $\Delta_{K M R}\left(t, \mu^{2}\right)=\sqrt{t} /\left(\sqrt{\mu^{2}}+\sqrt{t}\right)$ is the KMR cutoff function [11], and we have taken into account that $\mu^{2} \sim M_{T Y}^{2}$. The collinear singularity is regularized by the Sudakov formfactor:

$$
T_{i}\left(t, \mu^{2}\right)=\exp \left[-\int_{t}^{\mu^{2}} \frac{d t^{\prime}}{t^{\prime}} \frac{\alpha_{s}\left(t^{\prime}\right)}{2 \pi} \sum_{j=q, \bar{q}, g} \int_{0}^{1} d z z \cdot P_{j i}(z) \theta\left(1-\Delta_{K M R}\left(t^{\prime}, \mu^{2}\right)-z\right)\right],
$$

which resums doubly-logarithmic corrections $\sim \log ^{2}\left(t / \mu^{2}\right)$ in the LLA.

The final form of our unPDF in PRA is:

$$
\Phi_{i}\left(x, t, \mu^{2}\right)=\frac{T_{i}\left(t, \mu^{2}\right)}{t} \frac{\alpha_{s}(t)}{2 \pi} \sum_{j=q, \bar{q}, g} \int_{x}^{1} d z P_{i j}(z) \cdot \frac{x}{z} f_{j}\left(\frac{x}{z}, t\right) \cdot \theta\left(1-\Delta_{K M R}\left(t, \mu^{2}\right)-z\right),
$$

which coincides with Kimber, Martin and Ryskin (KMR) unPDF [11]. The KMR unPDF is actively used in the phenomenological studies employing $k_{T}$-factorization, but to our knowledge, the derivation, presented in [6] is the first systematic attempt to clarify it's relationships with MRK limit of the QCD amplitudes.

In contrast to most of studies in the $k_{T}$-factorization, the gauge-invariant matrix elements with off-shell initial-state partons (Reggeized quarks and gluons) from Lipatov's EFT [8, 9] allow one to study arbitrary processes involving non-Abelian structure of QCD without violation of SlavnovTaylor identities due to the nonzero virtuality of initial-state partons. This approach, together with KMR unPDF gives stable and consistent results in a wide range of phenomenological applications, which include the description of the angular correlations of dijets [5], $b$-jets [12], charmed [13] and bottom-flavored [6] mesons, as well as some other examples.

\section{$3 \quad$ Model for $\Upsilon D$ pair production}

Considering the inclusive production of $\Upsilon(1 S)+D$ meson pairs, one should take into account both direct and feeddown production of $\Upsilon(1 S)$. In the direct case, and taking into account the possibility 
of $g \rightarrow D$ and $c \rightarrow D$ fragmentation, one has the following LO subprocesses in PRA:

$$
\begin{aligned}
& R+R \rightarrow \Upsilon(1 S)+g(\rightarrow D), \\
& R+R \rightarrow \Upsilon(1 S)+c(\rightarrow D)+\bar{c} .
\end{aligned}
$$

The feed-down contribution includes decays of higher $S$-wave and $P$-wave states:

$$
\begin{aligned}
& R+R \rightarrow \Upsilon(2 S, 3 S)+g(\rightarrow D), \quad R+R \rightarrow \Upsilon(2 S, 3 S)+c(\rightarrow D)+\bar{c}, \\
& R+R \rightarrow \chi_{b}(2 P, 1 P)+g(\rightarrow D), \quad R+R \rightarrow \chi_{b}(2 P, 1 P)+c(\rightarrow D)+\bar{c} .
\end{aligned}
$$

According to NRQCD factorization formalism [1] final heavy quarkonium can be produced via colorsinglet and color-octet intermediate states. We will use the set of color-singlet and color-octet nonperturbative matrix elements, which has been obtained in Ref. [14] from the fit of inclusive $p_{T}$-spectra of $\Upsilon(n S)$, measured at the the LHC.

To describe the $D$-meson production we use the fragmentation model in which the transition of gluon or $c$-quark to the $D$-meson is described by corresponding fragmentation function $(\mathrm{FF})$ $D_{c, g}\left(z, \mu^{2}\right)$. We use universal scale-dependent $c$-quark and gluon fragmentation functions fitted to $e^{+} e^{-}$-annihilation data from CERN LEP1, in the Ref. [15]. The same FFs has been used earlier in the description of $D D$-pair production, measured by the LHCb collaboration [4].

\section{Results and discussion}

Here we discuss our results obtained for prompt $\Upsilon(1 S) D^{0,+}$ pair production in $p p$-collisions at $\sqrt{S}=7$ $\mathrm{TeV}$. Due to the lack of space, in the figures below, normalized spectra are plotted only for $\Upsilon(1 S) D^{0}$ production. The results for $\Upsilon(1 S) D^{+}$are similar. In the Fig. 1 we demonstrate, that transverse momentum and rapidity spectra of $\Upsilon(1 S)$ and $D^{0}$ are described well in the LO of PRA. We set the renormalization and factorization scales to $\mu_{R}=\mu_{F}=\frac{\xi}{2}\left(\sqrt{M_{\Upsilon}^{2}+p_{T \Upsilon}^{2}}+\sqrt{M_{D}^{2}+p_{T D}^{2}}\right)$ where $\xi=1$ for the central lines of our predictions, and we vary $1 / 2<\xi<2$ to estimate the scale-uncertainty of our prediction, which is shown in the figures by the gray band.

In the Fig. 2, we plot spectra for the following correlation variables: $M_{i n v}$ is the invariant mass of $\Upsilon D$ pair, $\triangle y=\left|y_{\Upsilon}-y_{D}\right|$ is the rapidity difference, $\Delta \phi=\left|\phi_{\Upsilon}-\phi_{D}\right|$ is the azimuthal angle difference, $A_{T}=\left(p_{T \Upsilon}-p_{T D}\right) /\left(p_{T \Upsilon}+p_{T D}\right)$ is the transverse momentum asymmetry. Theoretical predictions for $M_{i n v}, \triangle y$ and $A_{T}$ spectra agree well with experimental data. The $\triangle \phi$ spectrum in LO of PRA has typical shape for this kind of spectra. It has one peak at the $\triangle \phi \sim \pi$ and plateau at the $\triangle \phi \leq \pi / 2$. However, the experimental data from LHCb Collaboration, though having large errors, demonstrate existence of the second peak near the value $\triangle \phi \simeq 0$. This feature of the data remains unexplained.

Below we estimate relative contributions of different production mechanisms in the calculated total cross section $\left(\mathcal{B}_{\Upsilon(1 S) \rightarrow \mu^{+} \mu^{-}} \times \sigma^{\Upsilon D^{0}}\right)$, which is about $50 \mathrm{pb}$. Feeddown contribution from decays of higher bottomonium states $(2 S, 3 S, 2 P)$ is small, about $20 \%$. In the direct $\Upsilon(1 S) D^{0}$ channel, the gluon to $D$-meson fragmentation (4) dominates over $c$-quark fragmentation (5), $40 \mathrm{pb}$ and $5 \mathrm{pb}$, correspondingly. There are color-singlet $\left(b \bar{b}\left[{ }^{3} S_{1}^{(1)}\right]\right)$ and color-octet $\left(b \bar{b}\left[{ }^{3} S_{1}^{(8)}\right]\right)$ contributions in the the subprocess (4), the last one is dominant and gives about $30 \mathrm{pb}$. Having in mind that uncertainty of our calculation from choice of hard scale and color-octet matrix elements is about factor 2, we found that SPS contribution, calculated in the PRA, can account for approximately one half of experimental measured value of cross-section $\left(\mathcal{B}_{\mu^{+} \mu^{-}} \times \sigma_{\exp }^{\Upsilon D^{0}}\right)=155 \pm 21 \pm 7 \mathrm{pb}$. Such a way, contrary to the conclusion of Ref. [2], the contribution of DPS production mechanism is not dominant in the associated $\Upsilon(1 S) D$ production.

\section{Acknowledgments}

Authors thank the Ministry of Education and Science of the Russian Federation for financial support in the framework of the Samara University Competitiveness Improvement Program among the 
world's leading research and educational centers for 2013-2020, the task number 3.5093.2017/8.9. The work of A.K. and M.N. is supported in part by the Russian Foundation for Basic Research through the Grant No. MK 18-32-00060. A.K. and M.N. thank the II Institute for Theoretical Physics of Hamburg University for hospitality and personally, Prof. B.A. Kniehl and Dr. Zhi-Guo He for stimulating discussions.

\section{References}

[1] Bodwin G.T., Braaten E., Lepage G.P. Rigorous QCD analysis of inclusive annihilation and production of heavy quarkonium // Phys. Rev. 1995. V. D51. P. 1125.

[2] Aaij R. et al. [LHCb Collaboration]. Production of associated $\Upsilon$ and open pcharm hadrons in pp collisions at $\sqrt{S}=7$ and $8 \mathrm{TeV}$ via double parton scattering // J. High Energy Phys. 2016. V. 07. P. 052.

[3] Aaij R. et al. [LHCb Collaboration]. Measurements of prompt charm production cross-sections in pp collisions at $\sqrt{S}=13 \mathrm{TeV} / /$ J. High Energy Phys. 2016. V. 03. P. 159.

[4] Karpishkov A., Saleev V., and Shipilova A. Large-pT production of D mesons at the LHCb in the parton Reggeization approach // Phys. Rev. 2016. V. D94. P. 114012.

[5] Nefedov M.A., Saleev V.A., Shipilova A.V. Dijet azimuthal decorrelations at the LHC in the parton Reggeization approach // Phys. Rev. 2013. V. D87. P. 094030.

[6] Nefedov M.A., Karpishkov A.V., Saleev V.A.B $\bar{B}$ angular correlations at the LHC in the parton Reggeization approach merged with higher-order matrix elements // Phys. Rev. 2017. V. D96. P. 096019.

[7] Nefedov M.A., Saleev V.A. On the one-loop calculations with Reggeized quarks // Mod.Phys.Lett. 2017. V. A32. P. 1750207.

[8] Lipatov L.N. Gauge invariant effective action for high energy processes in QCD // Nucl. Phys. 1995. V. B452. P. 369.

[9] Lipatov L.N., Vyazovsky M.I. Quasi-multi-Regge processes with a quark exchange in the tchannel // Nucl. Phys. 2001. V. B597. P. 399.

[10] Antonov E.N., Lipatov L.N., Kuraev E.A., Cherednikov I.O. Feynman rules for effective Regge action // Nucl. Phys. 2005. V. B721. P. 111.

[11] Kimber M.A., Martin A.D., Ryskin M.G. Unintegrated parton distributions // Phys. Rev. 2001. V. D63. P. 114027.

[12] Saleev V.A., Shipilova A.V. Inclusive b-jet and bb-dijet production at the LHC via Reggeized gluons // Phys. Rev. 2012. V. D86. P. 034032.

[13] Maciula R, Saleev V.A., Shipilova A.V., Szczurek A. New mechanisms for double charmed meson production at the LHCb // Phys. Lett. 2016. V. B758. P. 458.

[14] Nefedov M.A., Saleev V.A., Shipilova A.V. Prompt $\Upsilon(n S)$ production at the LHC in the Regge limit of QCD // Phys. Rev. 2013. V. D88. P. 014003.

[15] Kniehl B.A., Kramer G., Schienbein I, Spiesberger H. Reconciling open-charm production at the Fermilab Tevatron with QCD // Phys. Rev. Lett. 96, 012001 (2006). 

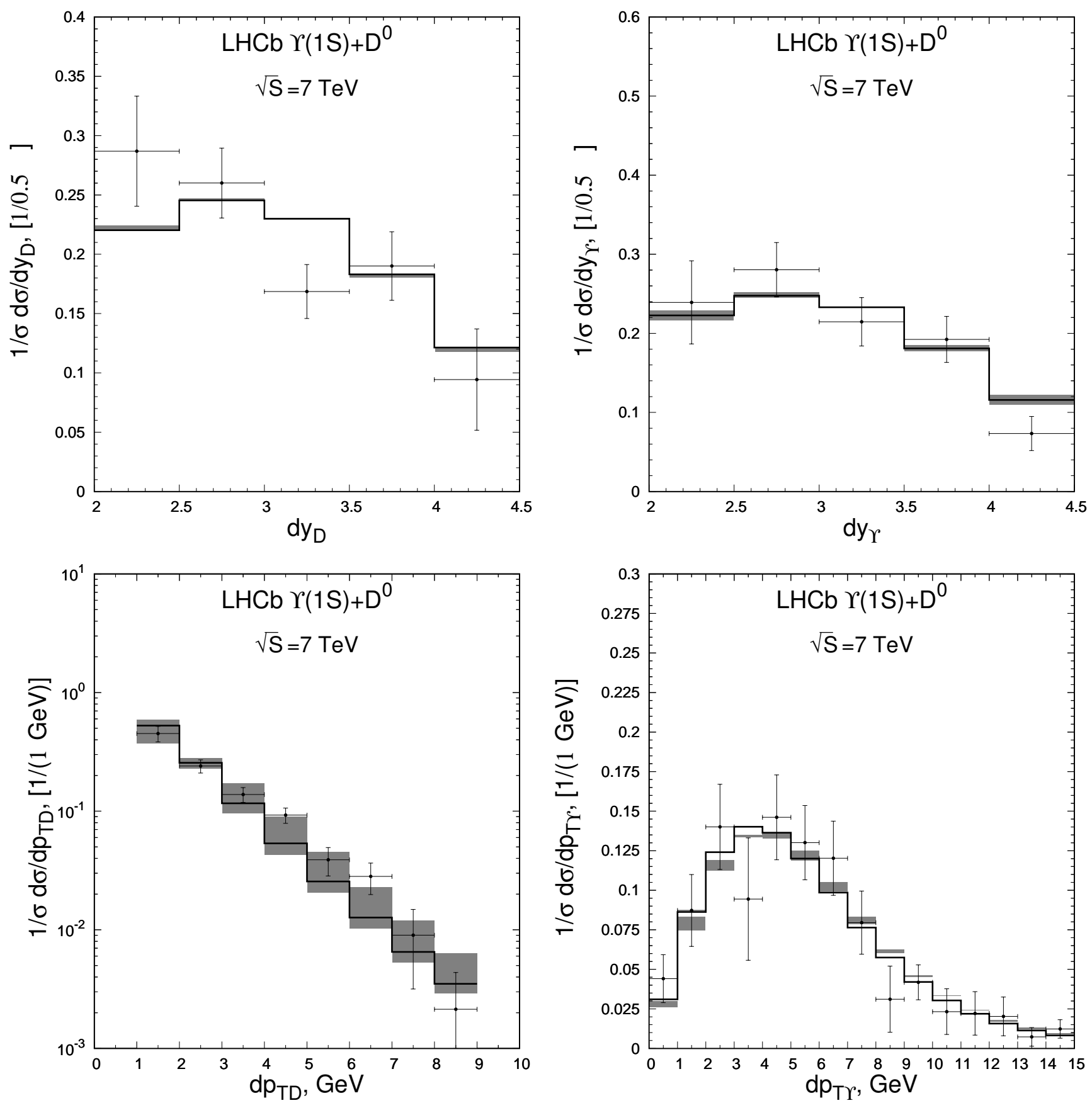

Figure 1: Transverse momentum and rapidity spectra of $\Upsilon(1 S)$ and $D^{0}$ in the associated production. The data from LHCb at the $\sqrt{S}=7 \mathrm{TeV}, 2.0<y_{\Upsilon(D)}<4.5,0<p_{T \Upsilon}<15 \mathrm{GeV}$, and $1<p_{T D}<20$ $\mathrm{GeV}$. 

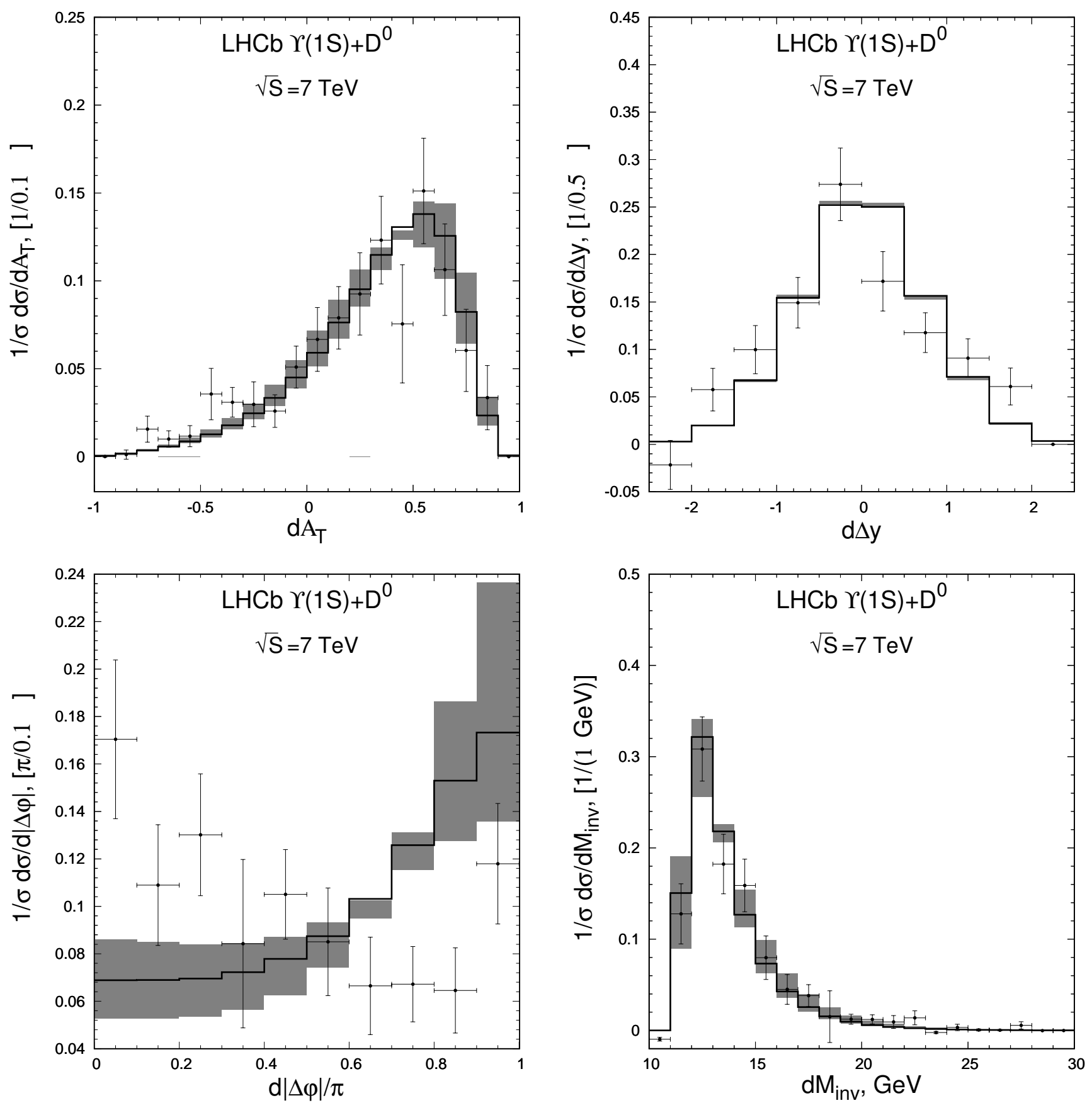

Figure 2: Spectra of $\Upsilon(1 S)+D^{0}$ associated production. The data from LHCb at the $\sqrt{S}=7 \mathrm{TeV}$, $2.0<y_{\Upsilon(D)}<4.5,0<p_{T \Upsilon}<15 \mathrm{GeV}$, and $1<p_{T D}<20 \mathrm{GeV}$. 